\title{
Preparación y Propiedades Fisicoquímicas de la N1-Acetilsulfanilamida de Hierro(III)
}

\author{
María C. Bordier, Juana A. de Morán, Ercilia A. Navarro y María Inés Gómez \\ Cátedras: Química Inorgánica y Química Analítica, Facultad de Bioquímica, Química y Farmacia, \\ Universidad Nacional de Tucumán, Ayacucho 491, (4000) San Miguel de Tucumán-Argentina \\ (e-mail: ccbordier@fbqf.unt.edu.ar)
}

\begin{abstract}
Resumen
En el presente trabajo se preparó mediante una técnica convencional de precipitación la sal de hierro(III) de la N1-Acetil sulfanilamida, también llamada sulfacetamida de hierro(III). Esto tiene como objetivo estudiar sus propiedades fisicoquímicas y proponer eventuales aplicaciones de la misma. Se determinó la composición, densidad, área superficial específica, solubilidad, constante del producto de solubilidad y los parámetros de envejecimiento del compuesto obtenido. Se estudió el proceso de descomposición térmica de la sustancia mediante análisis térmico gravimétrico (TGA) y térmico diferencial (DTA), en atmósfera de oxígeno. Los productos intermedios se identificaron utilizando espectroscopia infrarroja y análisis químicos. El proceso transcurre en varias etapas y el producto final es óxido de hierro(III), que se forma a temperaturas relativamente bajas. Esto sugiere la posible aplicación de los derivados metálicos de la sulfacetamida como precursores para la obtención de óxidos.
\end{abstract}

Palabras clave: sulfonamidas, N1-acetil sulfanilamida, iron salts, descomposición térmica

\section{Preparation and Physicochemical Properties of Iron (III) Salt of the N1-Acetyl Sulfanilamide}

\begin{abstract}
In the present work the iron (III) salt of the N1-acetyl sulfanilamide, know as iron (III) sulfacetamide, was prepared by using a conventional precipitation technique. This was done with the purpose of studying their physicochemical properties and to propose eventual applications of the same one. The composition, density, superficial area, solubility, constant of the solubility product, and aging parameters of the compound were determined. The thermal decomposition process was studied using thermo gravimetric (TGA) and differential thermo gravimetric (DTA) analysis in oxygen atmosphere. Infrared spectroscopy and chemical analysis were used to identify intermediate products. The process occurs at various stages, and the final product is iron(III) oxide that is formed at relatively low temperatures. This suggests the application of metallic derivates of sulfacetamide to obtain oxides.
\end{abstract}

Keywords: sulfonamide, N1-acetyl sulfanilamide, iron salts, thermal decomposition 


\section{INTRODUCCION}

Las sulfonamidas, sulfamidas o sulfas son sustancias sintéticas derivadas de la sulfanilamida o p-aminobenceno sulfonamida.

El descubrimiento de las sulfonamidas como antimicrobianos se debe a las investigaciones de Domagk que le valieron el premio Nobel en Medicina y Fisiología en 1939 (Velasco et al,1993). El compuesto original era un colorante y a partir de allí se sintetizaron numerosos derivados. Se prepararon más de 3300 sulfonamidas, de las cuales pocas alcanzaron utilidad clínica. (Litter,1982).

Además de la actividad antibacteriana en humanos, las sulfas se han usado en animales productores de alimentos, para promover su crecimiento y con fines terapéuticos y profilácticos. (Dixon y Katz,1991; Wen et al, 2005) Sin embargo se ha limitado su empleo debido a que los residuos de sulfas encontrados en la leche de algunos animales, son cancerígenos. Por esta razón la Unión Europea (EU) ha establecido el límite máximo de residuos en leche (MRL: Regulación $\mathrm{EC} / 281 / 96)$

Existen muchas áreas de potencial interés para la química de coordinación de las sulfonamidas, tales como la inhibición de la anhidrasa carbónica y actividad superoxidodismutasa, según Alzuet (1999) y Casanova et al. (2000). También Blasco et al (1996) y Chufán et al (1997), estudiaron la síntesis y las propiedades fisicoquímicas de complejos de sulfonamidas de metales de transición.

En el presente trabajo se informa sobre la preparación y propiedades fisicoquímicas de la sal de hierro(III) de la N1-Acetil sulfanilamida o sulfacetamida. El interés por estudiar el comportamiento del hierro(III) frente a las sulfas, radica en que la mayoría de las investigaciones anteriores se refieren a iones divalentes. Además la sulfa sintetizada, posiblemente podría tener aplicaciones terapéuticas tópicas.

\section{PARTE EXPERIMENTAL}

\section{Instrumental}

Analizador Termogravimétrico Shimadzu TG50; Analizador Termodiferencial Shimadzu DTA-50; Balanza Mettler Toledo Modelo AG 245- Precisión: 0,1mg; Autoanalizador
VITROS 250 - Química seca; Espectrofotómetro Metrolab 1600 PLUS-Precisión de lectura: Abs:0,0001 Transm: $99.97 \%$; Espectrofotómetro Infrarrojo de Transformada de Fourier (FTIR) Perkin Elmer 1600; Horno Mufla Thermolyne furnace-Type 48.000 y Microscopio electrónico de barrido Jeol JSM-35 CF. LAMENOA (CONICET-UNT).

\section{Reactivos}

Sulfacetamida sódica - USP - Merck Cloruro de hierro(III). $6 \mathrm{H}_{2} \mathrm{O}$ - Merck - p.a

Síntesis de sulfacetamida de hierro(III)

La preparación de este compuesto se realizó, a temperatura ambiente, empleando métodos clásicos de precipitación (Acevedo, 2001). Se agregó gota a gota y agitando, una solución de 2,70 g (10 mmoles) de cloruro de hierro(III) en $100 \mathrm{~mL}$ de agua bidestilada sobre una solución de 7,62 g (30 mmoles) de N1Acetilsulfanilamida de sodio en $100 \mathrm{~mL}$ de agua bidestilada. Se obtuvo un abundante precipitado pardo amarillento, que fue filtrado por papel de filtro para cristales finos, Wathman $\mathrm{N}^{\circ} 42$, lavado con agua bidestilada hasta ausencia de hierro(III), y secado en estufa a $100{ }^{\circ} \mathrm{C}$. El peso del sólido obtenido correspondía al $95 \%$ del rendimiento teórico.

\section{Composición del precipitado}

Para determinar la composición del precipitado se lo disolvió en $\mathrm{HCl}$ concentrado y luego se diluyó con agua. Se valoró la sulfacetamida mediante un proceso de diazotación (Farmacopea Nacional Argentina, 2000; US Pharmacopeia , 1995).

Fue necesario enmascarar el catión $\mathrm{Fe}(\mathrm{III})$ con $\mathrm{H}_{3} \mathrm{PO}_{4}$, para evitar la interferencia de color. El precipitado contenía $91,65 \%$ de sulfacetamida. El contenido de $\mathrm{Fe}$ (III) $(8,30 \%)$ del compuesto se determinó mediante la técnica espectrofotométrica de la o-fenantrolina, consultada en Skoog et al., (1997).

\section{Envejecimiento del precipitado}

El compuesto, preparado como se describió previamente, fue dejado en contacto con agua bidestilada, a temperatura ambiente, durante 210 días. A determinados intervalos de tiempo (tabla 1, fig.1), se extrajeron muestras para analizar Fe(III). Después de los 210 días se separó el sólido de igual manera que el precipitado reciente. 
Tabla 1: Valores de pKps y In C para distintos tiempos de envejecimiento

\begin{tabular}{|c|c|c|}
\hline Tiempo(días) & pKps & In c \\
\hline 3 & 14,62 & $-9,01$ \\
\hline 15 & 15,34 & $-9,56$ \\
\hline 30 & 16,18 & $-10,13$ \\
\hline 40 & 16,88 & $-10,80$ \\
\hline 70 & 17,75 & $-11,44$ \\
\hline 120 & 18,47 & $-12,19$ \\
\hline 150 & 18,60 & $-12,86$ \\
\hline 180 & 18,65 & $-13,56$ \\
\hline 210 & 18,60 & $-14,02$ \\
\hline
\end{tabular}

Para el precipitado reciente y el envejecido se determinó densidad, solubilidad y área superficial específica. (tabla 2)

\section{Densidad}

Se determinó para el precipitado reciente y el envejecido, a $25^{\circ} \mathrm{C}$, por un método picnométrico, utilizando $\mathrm{CCl}_{4}$ como medio líquido. Los resultados figuran en la tabla 2 .

\section{Solubilidad}

El compuesto recientemente preparado se suspendió en $100 \mathrm{~mL}$ de agua bidestilada, se agitó en forma continua durante 10 minutos a temperatura ambiente y se dejó en reposo 72 horas, para asegurar el equilibrio de disolución. La fase sólida no disuelta se separó por filtración con papel de poro fino.

Se determinó Fe(III) en el sobrenadante. Para ello se usaron dos técnicas diferentes, cuyos resultados se compararon: a) Una técnica de química seca, por medición de reflectancia. (Bender,1992). b) La técnica clásica de la ofenantrolina. A partir de los datos obtenidos, se calcularon los valores de la solubilidad, Kps y pKps del compuesto en estudio. Los resultados (tabla 2 ) fueron concordantes en ambos métodos.

\section{Área superficial específica}

A fin de evaluar el tamaño aproximado de partícula se determinó el área superficial específica de la muestra reciente y de la envejecida, mediante la técnica propuesta por Giles y Trivedi (1969), usando azul de metileno como adsorbente. Los resultados se detallan en la tabla 2. Del análisis de la variación de las concentraciones con el tiempo, se pudo conocer la velocidad de envejecimiento de la sulfacetamida de hierro(III). Para ello se realiza- ron las gráficas de $-\ln C, 1 / C$ y $1 / C^{2}$ frente al tiempo de envejecimiento. Se encontró una respuesta lineal para el primer caso, de donde se deduce que el proceso de envejecimiento, responde a una cinética de $1^{\text {er }}$ orden con respecto a la concentración de sulfacetamida de hierro(III). El valor de la constante cinética es de $1,53 \cdot 10^{-5} \mathrm{~s}^{-1}$.

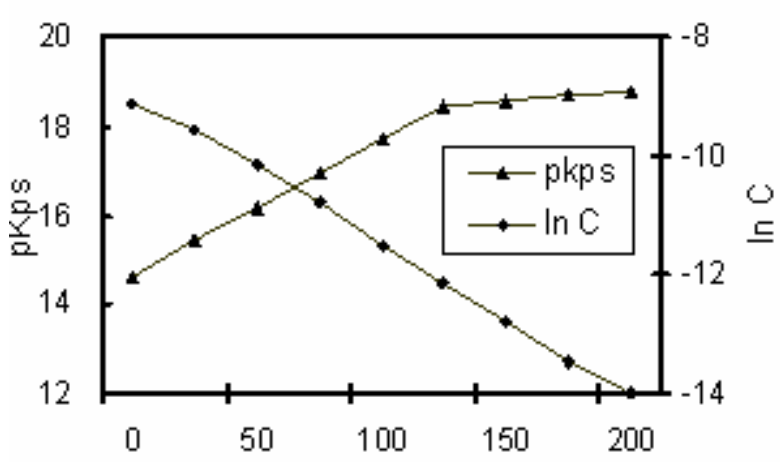

Fig. 1: pKps en función del tiempo de envejecimiento

Tabla 2: Propiedades físicas y químicas de la sulfacetamida de hierro (III)

\begin{tabular}{|c|c|c|c|}
\hline \multicolumn{2}{|c|}{ Propiedades } & Reciente & Envejecida \\
\hline \multicolumn{2}{|c|}{ Densidad (g/mL ) } & 1,623 & 1,658 \\
\hline \multirow{2}{*}{ 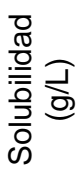 } & o-fenantrolina & $5,50.10^{-3}$ & $8,90 \cdot 10^{-6}$ \\
\hline & Química seca & $5,65 \cdot 10^{-3}$ & $8,95 \cdot 10^{-6}$ \\
\hline \multicolumn{2}{|c|}{$\begin{array}{c}\text { Área superficial } \\
\left(\mathrm{m}^{2} \mathrm{~g}^{-1}\right)\end{array}$} & 0,1520 & 0,0915 \\
\hline \multicolumn{2}{|c|}{$\mathrm{Kps}\left(\mathrm{mol}^{4} / \mathrm{L}^{4}\right)$} & $2,83.10^{-15}$ & $1,73.10^{-19}$ \\
\hline
\end{tabular}

\section{Microfotografías}

Se obtuvieron microfotografías del precipitado reciente y del envejecido, con un aumento de 200x (fig. 2 y 3, respectivamente). Del análisis de las mismas, se observa que, el envejecimiento del compuesto en presencia de las aguas madres, conduce a la formación de cristales aproximadamente cien veces mayores que los originales.

\section{Espectros de Infrarrojo}

Se realizó la pirólisis del compuesto en horno mufla para poder aislar los productos Intermedios y realizar los espectros de infrarrojo. 


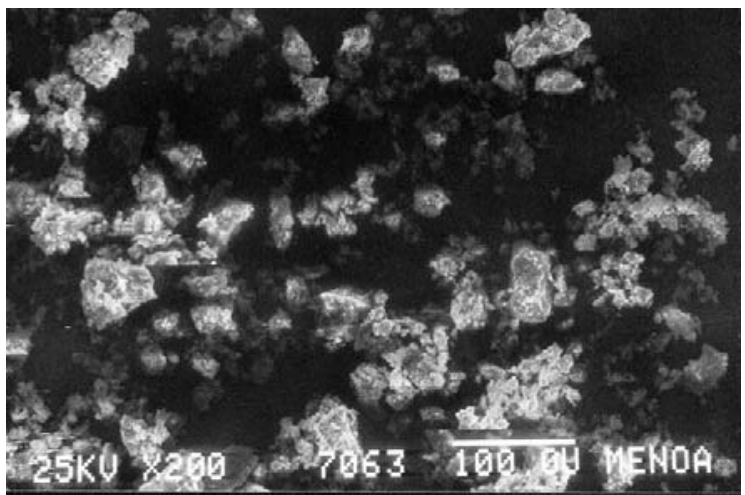

Fig.2: Microfotografía de una muestra reciente de sulfacetamida de hierro(III)

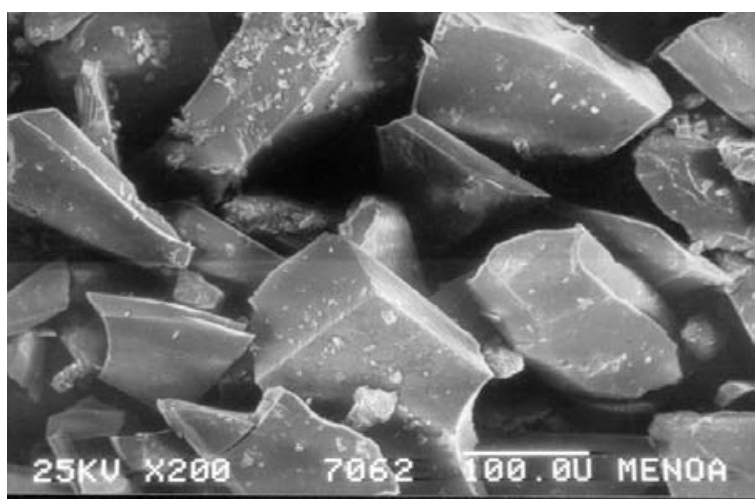

Fig. 3: Microfotografía de una muestra envejecida de sulfacetamida de hierro(III)

Los correspondientes al compuesto sintetizado y a los residuos obtenidos a $3500^{\circ} \mathrm{C}$ y 600 ${ }^{\circ} \mathrm{C}$, pueden observarse en las figuras 4,5 y 6 , respectivamente.

\section{Análisis térmico y térmico diferencial}

Los TGA y DTA se llevaron a cabo en atmósfera de oxígeno, con velocidad de calentamiento de $5^{\circ} \mathrm{C} / \mathrm{min}$ y flujo de gas de 30 $\mathrm{mL} / \mathrm{min}$ (fig. 7).

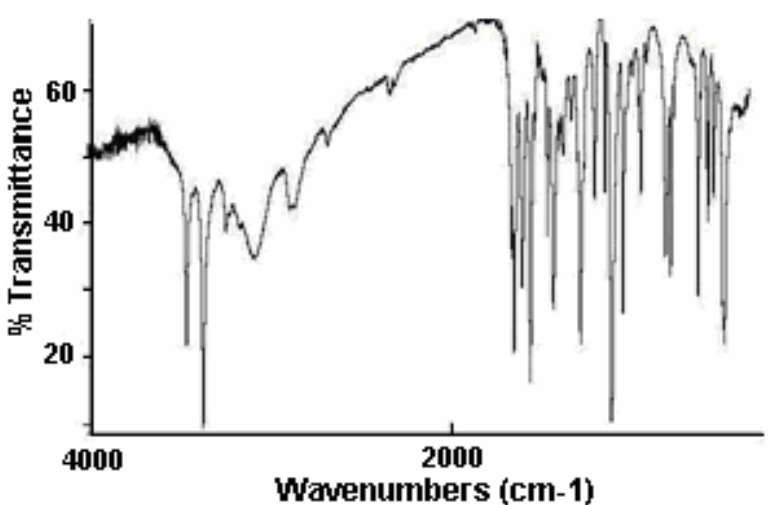

Fig.4: Espectro IR de la sulfacetamida de hierro(III)
Se observó que el residuo final a $600^{\circ} \mathrm{C}$, es negruzco, pero al estar en contacto con el aire se aclara de inmediato, tomando color pardo rojizo. Este cambio de color podría ser atribuido a la oxidación, por el oxígeno del aire, del hierro metálico formado a óxido de hierro(III) (Moeller, 1988).

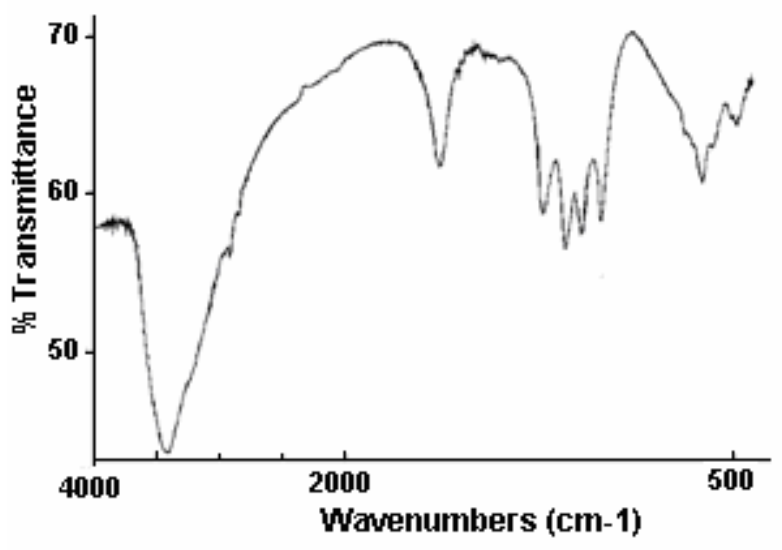

Fig.5: Espectro IR del residuo a $350^{\circ} \mathrm{C}$

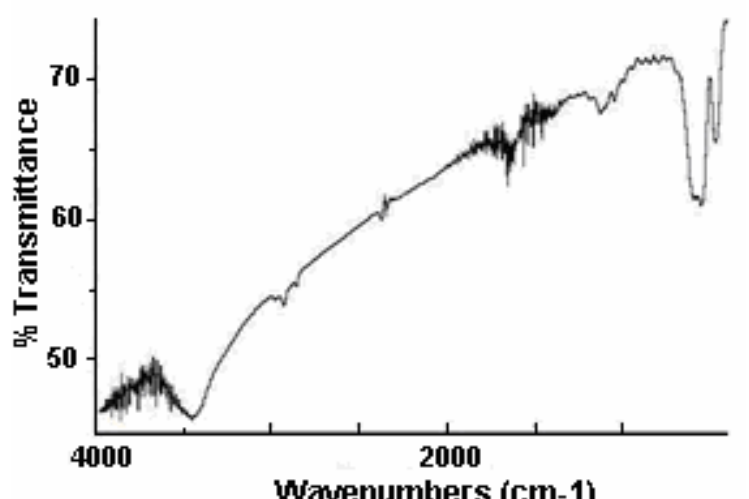

Fig. 6: Espectro IR del residuo a $600^{\circ} \mathrm{C}$

\section{RESULTADOS}

A través de los análisis químico y espectroscópico se constató que la sulfacetamida formaría un complejo con Fe(III) en relación molar 3:1. El mismo resultó muy poco soluble en agua de acuerdo a los valores de la Kps (tabla 2). Se encontró que la solubilidad disminuye linealmente en función del tiempo hasta aproximadamente 130 días, después de los cuales permanece constante. (fig. 1).

En la estructura del complejo, el átomo central es el $\mathrm{Fe}(\mathrm{III})$ que forma orbitales híbridos $d^{2} s p^{3}$ (Huheey,1981) lo que nos permite asignarle un índice de coordinación seis. Estos sitios de coordinación pueden ser ocupados por tres moléculas bidentadas del anión de la sulfacetamida. 


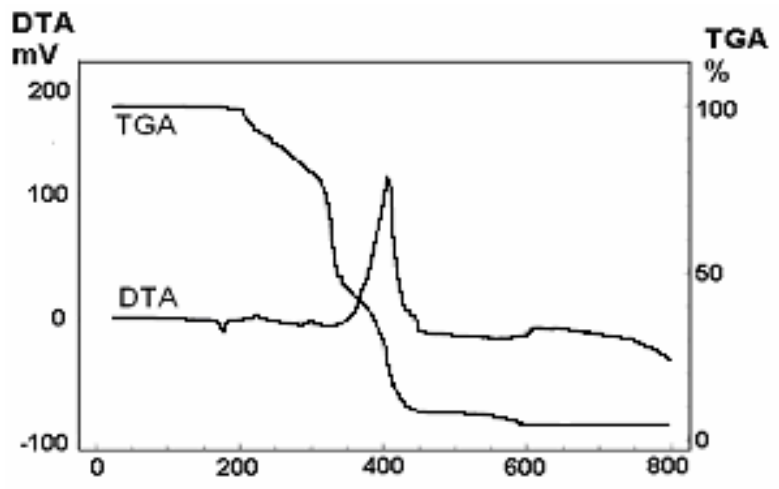

Fig.7: TGA y DTA de la sulfacetamida de hierro (III) en atmósfera de oxígeno

La unión del ligando al átomo central se efectuaría a través del oxígeno carbonilo del grupo acetilo y del oxígeno sulfonilo, formando anillos de seis miembros del tipo quelato. La disposición espacial más probable sería la octaédrica.(fig.8)

En el espectro infrarrojo (fig.4) aparecen las bandas características para los grupos sulfonilo, amino, bencénico y acetilo. En la sulfacetamida libre, las vibraciones de estiramiento atribuidas al grupo $-\mathrm{SO}_{2}$ - aparecen a 1320 , 1150 y $560 \mathrm{~cm}^{-1}$. En cambio cuando el sulfonilo es enlazado al $\mathrm{Fe}$ a través del átomo de oxígeno, el estiramiento antisimétrico será observado a $1325 \mathrm{~cm}^{-1}$ y el simétrico alrededor de $1140 \mathrm{~cm}^{-1}$. La evidencia de la deprotonación del grupo sulfacetamida y su coordinación a través del $O$ del grupo acetilo, surge del cambio de la banda asignada al grupo acetilo (1701 $\mathrm{cm}^{-1}$ en sulfacetamida libre y $1650 \mathrm{~cm}^{-1}$ en el complejo). (Smith, 1998) Como resultado del calentamiento del compuesto a $350{ }^{\circ} \mathrm{C}$, se produce la eliminación del grupo acetilo que se confirma por la desaparición en el espectro de IR de la banda ubicada a $1650 \mathrm{~cm}^{-1}$.

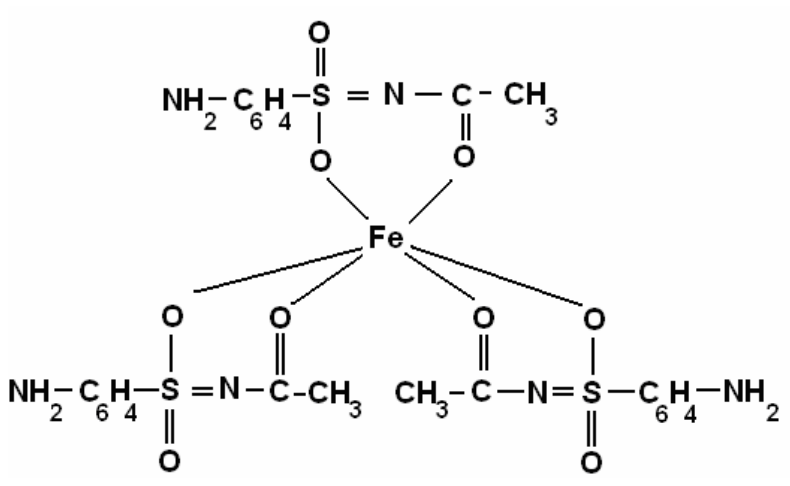

Fig. 8: Estructura de la sulfa de hierro(III)
Por otro lado, la presencia de las bandas típicas del grupo $-\mathrm{SO}_{2}$ - sugiere la posible formación de benceno sulfonato de hierro como producto intermedio. (fig.5). El producto final de la descomposición térmica a $600{ }^{\circ} \mathrm{C}$ corresponde al óxido de hierro(III) que verificamos con espectros de infrarrojo (fig.6) Silverstein y Webster (1996).

La curva termogravimétrica (TGA) (fig.7) denota que la sulfonamida de hierro(III) sintetizada es anhidra, ya que hasta $200^{\circ} \mathrm{C}$ el peso permanece constante. La descomposición se lleva a cabo en 4 etapas, con una pérdida de peso total de $95,72 \%$. Hasta $450{ }^{\circ} \mathrm{C}$ tiene lugar una importante pérdida de peso, (aproximadamente $90 \%$ respecto del peso inicial) que es mayor a la esperada. Por ello se podría suponer la formación de productos volátiles del tipo del hidruro de tetracarbonil hierro, que es un gas incoloro de estructura incierta, según Cotton y Wilkinson (1999).

En la curva termodiferencial (DTA) se observa un máximo endotérmico a $176,7^{\circ} \mathrm{C}$ y cuatro exotérmicos a $225,4{ }^{\circ} \mathrm{C}, 298,5^{\circ} \mathrm{C}, 406,6^{\circ} \mathrm{C}$ y $611,3^{\circ} \mathrm{C}$. El máximo endotérmico, podría corresponder a la fusión de la muestra o a un cambio de fase cristalográfica. Esto último está de acuerdo con estudios anteriores realizados sobre polimorfismo de las sulfonamidas (Wirth y Stephenson, 1997; Maury et al.1986). El máximo exotérmico de mayor superficie, ubicado a $406,6{ }^{\circ} \mathrm{C}$, se corresponde con la etapa de descomposición térmica del intervalo $350-420^{\circ} \mathrm{C}$. Esto está de acuerdo con la interpretación propuesta a partir del espectro IR a $350{ }^{\circ} \mathrm{C}$ (fig.5). La descomposición concluye a aproximadamente $600^{\circ} \mathrm{C}$ y el producto final es óxido de hierro(III).

\section{CONCLUSIONES}

De acuerdo a las observaciones realizadas, del análisis y discusión de los resultados, llegamos a las siguientes conclusiones sobre la nueva sulfonamida metálica sintetizada:

1) La sulfacetamida forma con Fe(III) un complejo, en relación 3:1.

2) Su preparación es simple y rápida usándose un método tradicional de precipitación.

3) El envejecimiento del producto sintetizado conduce a un importante crecimiento de los cristales. Ello explica la disminución de la solubilidad y del área superficial específica. 
4) Por acción de la temperatura, el producto sintetizado se descompone dejando como producto final óxido de hierro (III). Este hecho sugiere, la aplicación de la descomposición térmica de sulfonamidas metálicas, para la obtención de óxidos o mezclas de los mismos, a temperaturas relativamente bajas.

\section{REFERENCIAS}

Acevedo, V., M. Rodríguez y J. Giménez, "Estudio del proceso de precipitación de la 4amino $\mathrm{N}$-2 tiazolil bencenosulfonamida de Plomo (II)", Inf. Tecnológica, 12, 81-86 (2001).

Alzuet, D., "Coordinative versatility of the carbonic anhydrase inhibitor benzolamide in zinc and copper model compounds", Journal Inorganic Biochem.: 75,189-198 (1999).

Bender, G."Métodos instrumentales de análisis en química clínica" $1^{\text {a }}$ edición, 143-146 Acribia S.A, Zaragoza-España (1992).

Blasco, F., L. Perelló, J. Latorre, J.Borrás y S. Garciá-Granda."Cobalt(II), Nickel(II) and copper(II) complexes of sulfanilamide derivatives." Journal of Inorganic Biochemistry, 61,143-154 (1996).

Casanova, J., G.Alzuet, S. Ferrer, J. Latorre, J. Ramírez y J. Borrás, "Superoxide dismutase activity of ternary copper complexes of sulfathiazole and imidazole derivatives. Inorgánica Chímica Acta, 304,170-177 (2000).

Chufán, E., J. Pedregosa y J. Borrás, "Spectroscopic behaviour of metal-drug complexes" Vibrational Spectroscopy,15,191-199 (1997).

Cotton, F. y G. Wilkinson, "Química Inorgánica Básica", 10ª reimpresión, 540, Limusa S.A., México (1999).

Dixon Holland, D.E. y S.E. Katz, "Competitive Direct enzymelinked immunosorbent screening assay for the detection of sulfamethazine contamination of animal feeds", J. Assoc. Off. Anal. Chem., 74, 784-789 (1991).

Farmacopea Nacional Argentina, $7^{a}$ edición, Codex, Buenos Aires -Argentina (2000).

Giles, C.H. y A.S Trivedi., "Methods, apparatus: new product research, process development and design", Chemistry and Industry, 40, 1426-1427 (1969).
Huheey, J.,"Química Inorgánica",2a edición, capítulo 9, Harper \& Row Latinoamericana, D.F.México (1981).

Litter, M., "Compendio de Farmacología", 4a edición, Cap. 48: Las sulfonamidas y otros quimioterápicos: las diaminopirimidinas, El Ateneo, Buenos Aires, Argentina (1992).

Maury, L., J. Rambaud, B. Pauvert et al., "Etude physico-chimique de sulfamides: sulfadiazine, sulfadimethoxine et sulfamerazine",Farmaco Ed. Prat.,41,25 (1986).

Moeller, T. "Química Inorgánica" $10^{a}$ edición, 799- Reverté, Barcelona, España (1988).

MRL (Regulation EC/281/96). Norma de la European Union (EU) sobre el límite máximo de residuos de sulfonamidas en leche (1996).

Silverstein R., F.Webster,"Spectrometric identification of organic compounds", $6^{\mathrm{a}}$ edición,104 -107, John Wiley \& Sons, Inc. New York, United States of America (1996).

Skoog, D., D. West y F.Holler, "Química Analítica", 6ª edición, 604-605, McGraw -Hill, Guadalajara, México (1997).

Smith B.,"Infrared spectral interpretation",1 $1^{\mathrm{a}}$ edición, 138, 239, CRC Press, New York, United States of America (1998).

USPharmacopeia, 29rd edition, The United Status Pharmacopeial Convention, Rockville,MD (2006).

Velasco, A., P. Lorenzo y J. Serrano, "Farmacología Velásquez", $16^{\text {th }}$ edición, 894-901, Interamericana McGraw-Hill, Madrid, España (1993).

Wen, Y., M. Zhang, Q.Zhao y Y Feng, "Monitoring of five sulfonamide antibacterial residues in milk by in-tube solid-phase microextraction coupled to high-performance liquid chromatography", Q. J. Agric. Food Chem, 53, 8468-8473 (2005).

Wirth, D. y G. Stephenson, "Purification of dirithromycin. Impurity reduction and polymorph manipulation", Org. Process Res. Dev., 1, 55-60 (1997). 